\title{
Low-energy elastic electron scattering by acetaldehyde
}

\author{
A. Gauf, C. Navarro, G. Balch, L. R. Hargreaves, and M. A. Khakoo \\ Department of Physics, California State University, Fullerton, California 92834, USA \\ C. Winstead and V. McKoy \\ A. A. Noyes Laboratory of Chemical Physics, California Institute of Technology, Pasadena, California 91125, USA
}

(Received 6 January 2014; published 20 February 2014)

\begin{abstract}
We report results from a combined experimental and computational study of low-energy electron interactions with acetaldehyde in the gas phase. Differential cross sections for elastic electron scattering were measured at selected incident energies from 1 to $50 \mathrm{eV}$, while corresponding first-principles calculations were carried out up to $30 \mathrm{eV}$. Integral and momentum-transfer cross sections were derived from the angle-differential data. The role of resonances in the scattering is examined and comparison is made to previous results for acetaldehyde and for its analogs, formamide and formic acid.
\end{abstract}

DOI: 10.1103/PhysRevA.89.022708

PACS number(s): $34.80 . \mathrm{Bm}$

\section{INTRODUCTION}

Acetaldehyde or ethanal, $\mathrm{CH}_{3} \mathrm{CHO}$, is the simplest aldehyde apart from formaldehyde, $\mathrm{H}_{2} \mathrm{CO}$, from which it differs by the replacement of one $\mathrm{H}$ atom with a methyl group. Acetaldehyde was one of the first organic molecules identified in the interstellar medium [1-3]. Like formaldehyde and formic acid $(\mathrm{HCOOH})$, acetaldehyde has both a large permanent electric dipole moment and an empty carbonyl $\pi^{*}$ orbital that may trap electrons to form metastable states and so give rise to resonances in photoexcitation and electron scattering. The role of dipole-bound resonances, their evolution with bond length into $\sigma^{*}$ resonances, and the possible interplay between such resonant mechanisms and the $\pi^{*}$ resonance are subjects of considerable interest and some controversy [4-7]. Determining cross sections for elastic scattering of low-energy electrons by acetaldehyde is a step toward understanding such resonant processes.

We know of no prior measurements of the elastic-electronscattering cross section of acetaldehyde. However, van Veen and coworkers [8] and Jordan and Burrow [9] measured the transmitted electron current (or its derivative), which is sensitive to the presence of narrow elastic resonances. The expected C-O $\pi^{*}$ shape resonance was observed at $1.3 \mathrm{eV}$ [8] and at $1.19 \mathrm{eV}$ [9]. In an electron energy-loss experiment, Dressler and Allan [10] observed electronic Feshbach resonances between 6 and $7 \mathrm{eV}$. Measured cross sections for vibrational excitation by electron impact have shown both the $\pi^{*}$ resonance $[10,11]$, at about $1.2 \mathrm{eV}$, and a further broad peak at $6.8 \mathrm{eV}$, assigned to one or more $\sigma^{*}$ resonances [10]. Other measurements have determined thresholds and, in some cases, relative cross sections for electronic excitation $[8,10,12-16]$, while still others have examined dissociative electron attachment [10,17-19] and electron-impact ionization [20-22]. Burean and Swiderek [23] have studied the chemistry induced in condensed acetaldehyde by low-energy electron impact. Recently, Szmytkowski [24] reported absolute measurements of the total cross section (TCS) for electron scattering by acetaldehyde over an extensive energy range, from 0.7 to $400 \mathrm{eV}$. Although broad maxima are visible in the TCS between 5 and $10 \mathrm{eV}$, the $\pi^{*}$ resonance is not observed.

Acetaldehyde is isoelectronic with formamide, $\mathrm{NH}_{2} \mathrm{HCO}$, and the two are similar in both overall structure and dipole moment. Recent measurements [25-27] and calculations [2830] have studied low-energy resonant scattering or attachment processes in formamide and have provided some information on its elastic cross section [29-31]. Acetaldehyde is likewise isoelectronic with and structurally similar to formic acid, though formic acid has a significantly smaller dipole moment. Low-energy electron collisions with formic acid have been the subject of a number of recent experimental [7,32-40] and theoretical [4-6,41-45] studies. Given the dearth of data on elastic scattering by acetaldehyde itself, results for these two analogs are useful points of comparison.

The present paper reports a combined experimental and computational study of elastic scattering of low-energy electrons by acetaldehyde. Measurements of the differential cross section (DCS) were performed over a range of scattering angles and at energies from 1 to $50 \mathrm{eV}$, while calculations of the DCS were carried out from 0.1 to $30 \mathrm{eV}$. The integral cross section (ICS) was estimated from the measured and calculated DCSs after correcting for the strong scattering at near-forward angles due to the long-range potential created by the permanent electric dipole moment.

\section{EXPERIMENT}

The experimental apparatus has been described previously, e.g., by Khakoo and coworkers [46], so only a brief description is given here. The electron gun and the detector employ double hemispherical energy selectors with cylindrical electrostatic lenses, and the apparatus is made of titanium. The system was heated to about $130{ }^{\circ} \mathrm{C}$ with magnetically free biaxial heaters (ARi Industries Model BXX06B41-4K). The analyzer detector consisted of a discrete dynode electron multiplier (Equipe Thermodynamique et Plasmas Model AF151) with the extremely low background rate of $<0.01 \mathrm{~Hz}$ and the capability of linearly detecting up to $1 \mathrm{MHz}$ of electrons without saturating. The analyzer employed a virtual-apertures configuration downstream from the entrance (0.7- and 1-mm-diameter apertures), which enabled a more efficient transmission of low-residual-energy electrons (since these apertures could be operated at a higher energy, rather than being fixed at the collision region potential). A skimmer nose piece with a 3-mm aperture at the entrance of this analyzer served to suppress secondary electrons and also limited the 
depth of field of the detector to be $\pm 3 \mathrm{~mm}$ around the collision region. The remnant magnetic field in the collision region is reduced to about $1 \mathrm{mG}$ by using a double $\mu$-metal shield as well as a coil that eliminated the vertical component of Earth's magnetic field. Typical electron currents were around 20 to $25 \mathrm{nA}$, with an energy resolution of 50 to $60 \mathrm{meV}$, full width at half maximum. The electron beam could be focused at energies below $1.0 \mathrm{eV}$ and up to $100 \mathrm{eV}$. The spectrometer current remained stable to within $10 \%$ over a period of several weeks, requiring minor tuning of the spectrometer to maintain the long-term stability of the current to within $10 \%$ at any time. The energy of the beam was established by determining the dip in the He elastic-scattering cross section due to the $2^{2} S \mathrm{He}^{-}$resonance at $19.366 \mathrm{eV}$ [47] to an uncertainty of $\pm 20 \mathrm{meV}$ during a run at a given impact energy $E_{0}$. Typically the contact potential so determined drifted about the value of $0.95 \mathrm{eV}$ by $\pm 0.05 \mathrm{eV}$ over the multiweek course of the experiments. Energy-loss spectra of the elastic peak were collected at fixed incident electron energy $\left(E_{0}\right)$ values and electron-scattering angles $\theta$ by repetitive, multichannel-scaling techniques. The angular resolution was $2^{\circ}$, full width at half maximum. The effusive target gas beam was formed by flowing gas through a thin aperture source $0.3 \mathrm{~mm}$ in diameter described previously [48]. This source was sooted, using an acetylene flame, to reduce secondary electrons and placed $6 \mathrm{~mm}$ below the axis of the electron beam, incorporated into a movable source arrangement [49]. The movable gas source method has been well tested previously in our laboratory and determines background scattering rates expediently and accurately. The vapor pressure behind the source for acetaldehyde was between 0.15 and 0.2 Torr and that for helium was between 1.0 and 1.6 Torr, while the pressure in the experimental chamber was around $1 \times 10^{-6}$ Torr. The gas beam temperature, determined by the apparatus temperature in the collision region, was about $130{ }^{\circ} \mathrm{C}$; however, in most of the gas-handling copper tubing, the temperature was about $85^{\circ} \mathrm{C}$, with the higher temperature only in the last $4 \mathrm{~cm}$ of the tube before the gas exited into the collision region. Based on the flow rate vs drive pressure analysis [48], the gas-kinetic molecular diameter of acetaldehyde was determined to be $7.27 \times 10^{-8} \mathrm{~cm}$. The differential cross sections for elastic scattering from acetaldehyde were normalized via relative flow [48] to the well-established elastic cross sections for He from Ref. [50].

Our elastic-scattering measurements were taken at $E_{0}$ values of $1,2,3,5,10,15,20,30$, and $50 \mathrm{eV}$ for scattering angles ranging from $5^{\circ}$ to $130^{\circ}$. To compute integral elastic and momentum-transfer cross sections, the measured DCSs were extrapolated to $0^{\circ}$ and $180^{\circ}$ using a smooth polynomial fit. To account for the strong forward scattering due to the dipole moment, the ICS was also evaluated using the first Born approximation to the point-dipole DCS in the extrapolation, with a dipole moment of $2.72 \mathrm{D}$ [51] and an energy loss of $5 \mathrm{meV}$.

\section{COMPUTATION}

The calculations employed the Schwinger multichannel (SMC) method [52,53] as implemented for parallel computers [54,55]. General information about the SMC method may be found in the references cited, so here we describe only the details particular to the present study of acetaldehyde.
TABLE I. Centers $(x, y, z)(\AA)$ of the supplementary Gaussian basis functions used in the calculations on acetaldehyde. The supplement on the centers labeled $Q_{i}$ consisted of five $s$ Gaussians (exponents 0.1, 0.03, 0.01, 0.003, and 0.001) and two $p$ Gaussians (exponents 0.03 and 0.01 ), while a single $s$ Gaussian with exponent 0.07 was placed on the centers labeled $X_{n}$. The coordinates of the nuclei are also shown for reference.

\begin{tabular}{lccc}
\hline \hline Center & $x$ & $y$ & $z$ \\
\hline $\mathrm{O}$ & -1.1514 & -0.2322 & 0.0 \\
$\mathrm{C}$ & -0.1193 & 0.4248 & 0.0 \\
$\mathrm{H}$ & -0.1608 & 1.5332 & 0.0 \\
$\mathrm{C}$ & 1.2622 & -0.1680 & 0.0 \\
$\mathrm{H}$ & 1.1972 & -1.2565 & 0.0 \\
$\mathrm{H}$ & 1.8143 & 0.1751 & \pm 0.8814 \\
$Q_{1}$ & 3.0 & 1.36 & 0.0 \\
$Q_{2}$ & 6.0 & 2.72 & 0.0 \\
$X_{1}$ & \pm 3.0 & 0.0 & 0.0 \\
$X_{2}$ & 0.0 & \pm 3.0 & 0.0 \\
$X_{3}$ & 0.0 & \pm 3.0 \\
$X_{4}$ & \pm 3.0 & 0.0 & 0.0 \\
$X_{5}$ & \pm 3.0 & \pm 3.0 & \pm 3.0 \\
$X_{6}$ & 0.0 & \pm 3.0 & \pm 3.0 \\
$X_{7}$ & \pm 3.0 & 0.0 & \pm 3.0 \\
$X_{8}$ & \pm 6.0 & \pm 6.0 & 0.0 \\
$X_{9}$ & 0.0 & 0.0 & 0.0 \\
$X_{10}$ & 0.0 & & \pm 6.0 \\
\hline \hline
\end{tabular}

All calculations were carried out within the fixed-nuclei approximation using the ground-state equilibrium geometry of acetaldehyde as computed at the level of second-order Möller-Plesset perturbation theory within the 6-31G $(d)$ basis set $[56,57]$. The minimum-energy conformation belongs to the $C_{s}$ point group and has one of the methyl hydrogens $\mathrm{cis}$ to the carbonyl oxygen. The geometry optimization and subsequent bound-state electronic structure calculations were carried out with the program system GAMESS [58].

The choices of one-electron and many-electron basis sets to describe the electronic structure of the target molecule and the structure and dynamics of the electron-molecule collision complex are both important considerations in the SMC approach. Our one-electron basis for acetaldehyde incorporated a standard contracted Gaussian basis set, the augmented correlation-consistent polarized valence double- $\zeta$ (aug-ccpVDZ) set $[59,60]$, supplemented with uncontracted Gaussian functions distributed around the periphery of the molecule. The latter set included diffuse $s$ and $p$ Gaussians located at the positive end of the molecule as well as $s$ Gaussians centered at grid points on all sides of the molecule, as detailed in Table I. These added Gaussians improve the description of possible dipole-bound states and expand the "computational box" within which the scattering function is represented. Our experience has been that adding such functions improves the numerical stability of the calculation, provided of course that care is taken to avoid introducing linear dependence in the basis. After excluding the $x^{2}+y^{2}+z^{2}$ linear combinations of the $d$ Gaussians, the overall one-electron basis set contains 159 contracted Gaussians from which to construct the 12 doubly occupied Hartree-Fock orbitals describing the ground state of 
the target. It should be noted that, despite using an extended and flexible basis set, we obtained a value of $3.347 \mathrm{D}$ for the static electric dipole moment of the ground state, which is $22 \%$ larger than the experimental value, $2.750 \mathrm{D}$ [61]. It is necessary to go beyond the Hartree-Fock level to obtain a better value of the dipole moment; for example, even without the supplementary functions of Table I, the aug-cc-pVDZ basis yields a much more accurate value of $2.808 \mathrm{D}$ at the level of second-order Möller-Plesset perturbation theory.

The 147 unoccupied Hartree-Fock orbitals were subjected to an orthogonal transformation to form "modified virtual orbitals" [62], using a +6 cationic Fock operator, in order to generate a set of compact, valencelike orbitals suitable for use as particle orbitals when representing polarization and correlation through virtual excitations [63]. The SMC variational space of many-electron functions was then constructed from three classes of doublet configuration state functions. The first class consists of the ground-state Hartree-Fock wave function antisymmetrized with each of the virtual orbitals.
The second, and by far the largest, class consists of singlet single excitations from the 9 valence orbitals of the ground state into the 30 lowest-energy modified virtual orbitals, again coupled with all of the virtual orbitals to form doublet configuration state functions. The third class includes doublet functions formed from the triplet single excitation of the $\mathrm{C}-\mathrm{O}$ $\pi$ orbital into the lowest-energy virtual orbital, which has $\mathrm{C}-\mathrm{O}$ $\pi^{*}$ character, coupled with all virtual orbitals. These three classes are intended to describe, respectively, scattering from the unrelaxed target charge density, orbital relaxation during the collision ("polarization"), and virtual excitation to the low-lying $\left(\pi \rightarrow \pi^{*}\right) 1^{3} A^{\prime}$ state. It should be noted, however, that the second class also describes virtual excitation of the $\left(\pi \rightarrow \pi^{*}\right) 2{ }^{1} A^{\prime}$ state (and other singlet states).

Separate scattering calculations were carried out for the ${ }^{2} A^{\prime}$ and ${ }^{2} A^{\prime \prime}$ representations of the $C_{S}$ symmetry group. The respective SMC variational spaces contained 19147 and 16921 configuration state functions. The linear systems arising from the variational procedure were solved via
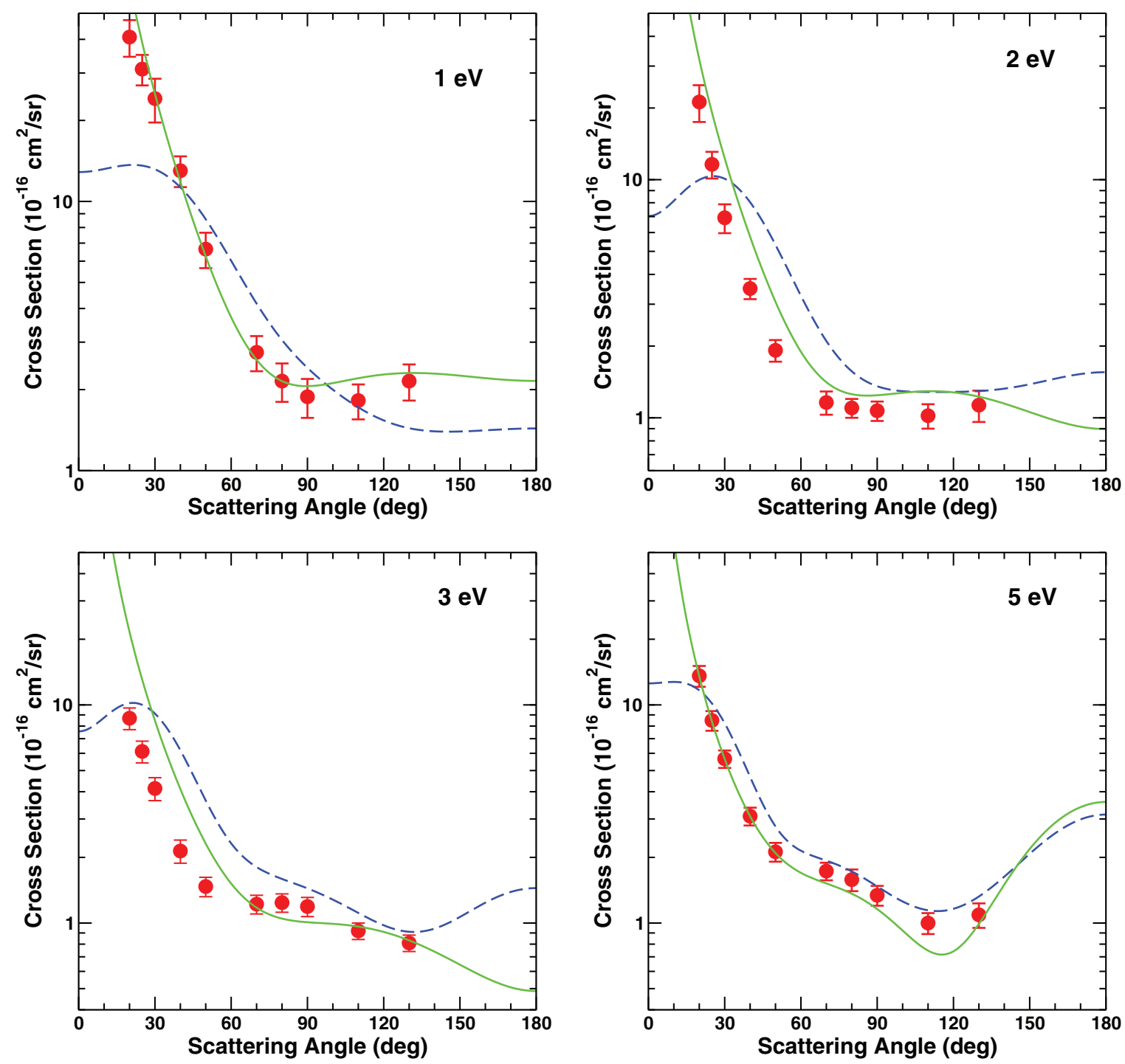

FIG. 1. (Color online) Differential cross section for elastic electron scattering by acetaldehyde at the collision energy indicated in each panel. Red circles are the present measurements, the blue dashed line is the present calculation, and the green solid line is the present calculation including the Born correction. 
singular-value decomposition, with one vector being deleted from the ${ }^{2} A^{\prime}$ space to improve numerical stability. The ${ }^{2} A^{\prime}$ and ${ }^{2} A^{\prime \prime}$ scattering amplitudes obtained from the solutions of the linear systems were summed to obtain the physical scattering amplitude. A correction for long-range scattering due to the dipole potential was then applied, using a form of the standard "Born completion" procedure [64]. In this procedure, the contributions of low partial waves to the scattering amplitude are taken from the SMC calculation, while those of higher waves are obtained from the first Born approximation for a fixed-point dipole. To be consistent with the description of the target molecule used in the SMC calculation, we used the Hartree-Fock dipole moment in this step. The cutoff, denoted $L_{\max }$, for the angular momenta retained from the SMC calculation was decided by a semiclassical criterion, $R p \approx$ $\hbar\left[L_{\max }\left(L_{\max }+1\right)\right]^{\frac{1}{2}}$, where $R$, the radius of the molecular charged density, is taken as approximately 4 bohr units and $p$ is the momentum of the projectile electron. The cutoffs chosen in rough consistency with this criterion were $L_{\max }=1$ up to $3 \mathrm{eV}, L_{\max }=2$ above 3 and up to $5 \mathrm{eV}, L_{\max }=3$ above 5 and up to $7.5 \mathrm{eV}, L_{\max }=4$ above 7.5 and up to $15 \mathrm{eV}, L_{\max }=5$
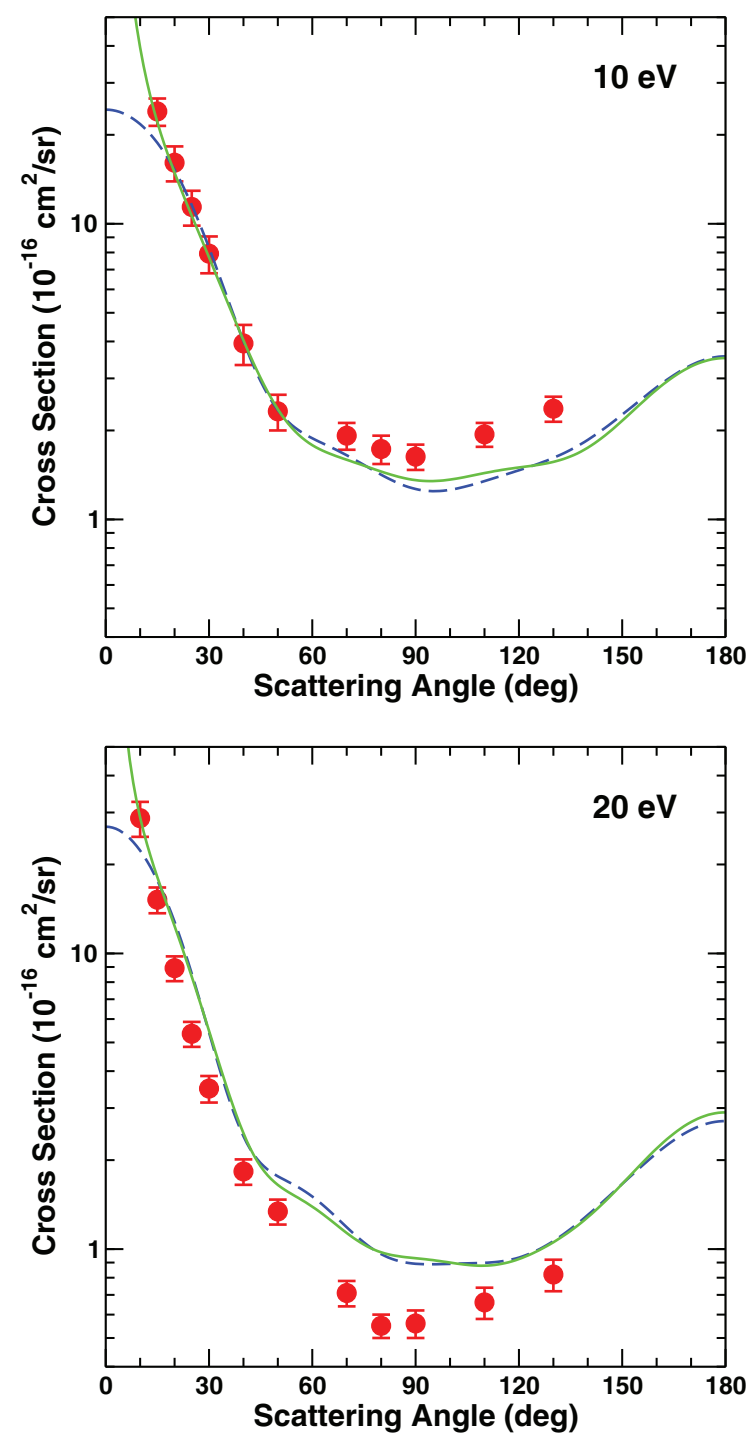

above 15 and up to $22 \mathrm{eV}$, and $L_{\max }=6$ above 22 and up to $30 \mathrm{eV}$.

Because the first-Born DCS for elastic scattering by a fixed-point dipole has a nonintegrable singularity at $0^{\circ}$, some inelasticity must be assumed to obtain a finite ICS. In the present work, we computed the ICS by combining the pure dipole-Born DCS for an energy loss of $0.01 \mathrm{eV}$, analytically integrated from $0^{\circ}$ to 2 , with the corrected DCS described above, numerically integrated from $2^{\circ}$ to $180^{\circ}$. The choice of $0.01 \mathrm{eV}$ for the inelasticity is arbitrary but intended to be representative of typical rotational transitions in room-temperature acetaldehyde; doubling or halving this value changes the resulting ICS by $\sim 5 \%$ or less, depending on the electron energy. The choice of matching angle is likewise arbitrary, and the results are likewise insensitive to modest variation of that choice.

\section{RESULTS AND DISCUSSION}

Differential cross sections for elastic electron scattering from acetaldehyde are shown at selected collision energies in Figs. 1 and 2, and the experimental values are listed in
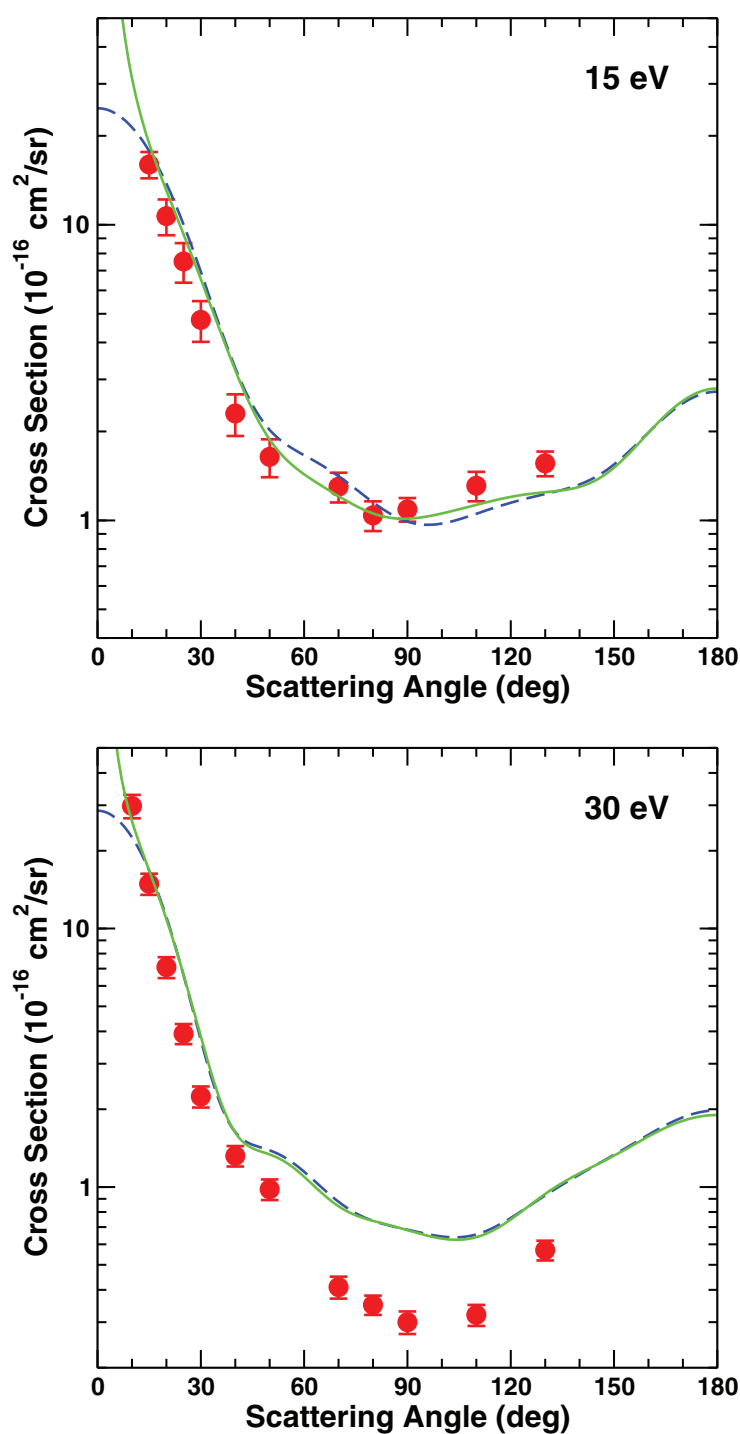

FIG. 2. (Color online) As in Fig. 1, at higher energies. 
TABLE II. Measured differential cross sections $\left(10^{-16} \mathrm{~cm}^{2} / \mathrm{sr}\right)$ for elastic electron scattering by acetaldehyde. The second column at each energy lists the error estimate. Italicized entries are extrapolated values used in computing the integral elastic (ICS) and momentum-transfer (MTCS) cross sections, which are listed, along with their error estimates, at the foot of the columns in $10^{-16} \mathrm{~cm}^{2}$ units. The notation $x(y)$ signifies $x \times 10^{y}$.

\begin{tabular}{|c|c|c|c|c|c|c|c|c|c|c|c|c|c|c|c|c|c|c|}
\hline \multirow{2}{*}{$\frac{\text { Angle (deg) }}{0}$} & \multicolumn{2}{|c|}{$1 \mathrm{eV}$} & \multicolumn{2}{|c|}{$2 \mathrm{eV}$} & \multicolumn{2}{|c|}{$3 \mathrm{eV}$} & \multicolumn{2}{|c|}{$5 \mathrm{eV}$} & \multicolumn{2}{|c|}{$10 \mathrm{eV}$} & \multicolumn{2}{|c|}{$15 \mathrm{eV}$} & \multicolumn{2}{|c|}{$20 \mathrm{eV}$} & \multicolumn{2}{|c|}{$30 \mathrm{eV}$} & \multicolumn{2}{|c|}{$50 \mathrm{eV}$} \\
\hline & $8.09(5)$ & & $2.27(6)$ & & $1.62(6)$ & & $7.60(6)$ & & $5.00(3)$ & & $4.00(3)$ & & $3.00(3)$ & & $3.00(3)$ & & $1.00(3)$ & \\
\hline 1 & $1.39(4)$ & & $9.84(3)$ & & $3.13(3)$ & & $5.28(3)$ & & $2.00(3)$ & & $1.00(3)$ & & 750 & & 600 & & 350 & \\
\hline 3 & $1.57(3)$ & & $1.10(3)$ & & 349 & & 587 & & 500 & & 300 & & 220 & & 160 & & 120 & \\
\hline 5 & 565 & & 395 & & 126 & & 211 & & 130 & & 110 & & 90.0 & & 85.0 & & 60.5 & 8.96 \\
\hline 8 & 221 & & 155 & & 49.1 & & 82.7 & & 55.0 & & 45.0 & & 42.0 & & 42.0 & & & \\
\hline 10 & 142 & & 99.1 & & 31.5 & & 53.0 & & 40.0 & & 30.0 & & 28.7 & 3.89 & 29.8 & 3.09 & 23.4 & 2.41 \\
\hline 15 & 63.1 & & 44.2 & & 14.0 & & 23.6 & & 24.0 & 2.55 & 16.0 & 1.63 & 15.2 & 1.52 & 14.9 & 1.40 & 9.65 & 0.94 \\
\hline 20 & 40.8 & 6.34 & 21.2 & 3.73 & 8.69 & 0.99 & 13.6 & 1.49 & 16.1 & 2.18 & 10.7 & 1.48 & 8.92 & 0.86 & 7.09 & 0.66 & 3.86 & 0.37 \\
\hline 25 & 31.0 & 4.02 & 11.6 & 1.49 & 6.12 & 0.70 & 8.49 & 0.88 & 11.4 & 1.54 & 7.52 & 1.15 & 5.35 & 0.52 & 3.92 & 0.35 & 1.84 & 0.23 \\
\hline 30 & 24.1 & 4.46 & 6.92 & 0.96 & 4.14 & 0.50 & 5.66 & 0.52 & 7.93 & 1.13 & 4.77 & 0.75 & 3.49 & 0.36 & 2.24 & 0.21 & 1.46 & 0.16 \\
\hline 40 & 13.0 & 1.70 & 3.49 & 0.34 & 2.14 & 0.26 & 3.09 & 0.29 & 3.94 & 0.61 & 2.30 & 0.37 & 1.83 & 0.18 & 1.32 & 0.12 & 0.84 & 0.09 \\
\hline 50 & 6.65 & 1.00 & 1.92 & 0.20 & 1.47 & 0.15 & 2.12 & 0.21 & 2.32 & 0.32 & 1.64 & 0.24 & 1.34 & 0.13 & 0.98 & 0.09 & 0.48 & 0.06 \\
\hline 70 & 2.75 & 0.41 & 1.16 & 0.13 & 1.22 & 0.12 & 1.73 & 0.16 & 1.92 & 0.20 & 1.30 & 0.15 & 0.71 & 0.07 & 0.41 & 0.04 & 0.19 & 0.02 \\
\hline 80 & 2.15 & 0.35 & 1.10 & 0.10 & 1.24 & 0.12 & 1.58 & 0.18 & 1.73 & 0.19 & 1.04 & 0.12 & 0.55 & 0.05 & 0.35 & 0.03 & 0.15 & 0.01 \\
\hline 90 & 1.88 & 0.31 & 1.07 & 0.10 & 1.19 & 0.12 & 1.34 & 0.14 & 1.63 & 0.16 & 1.09 & 0.10 & 0.56 & 0.06 & 0.30 & 0.03 & 0.13 & 0.02 \\
\hline 110 & 1.82 & 0.27 & 1.02 & 0.12 & 0.92 & 0.08 & 1.00 & 0.11 & 1.94 & 0.18 & 1.31 & 0.15 & 0.66 & 0.08 & 0.32 & 0.03 & 0.23 & 0.02 \\
\hline 130 & 2.15 & 0.33 & 1.13 & 0.17 & 0.81 & 0.07 & 1.09 & 0.14 & 2.37 & 0.23 & 1.56 & 0.15 & 0.82 & 0.10 & 0.57 & 0.05 & 0.27 & 0.03 \\
\hline 140 & 2.50 & & 1.40 & & 0.90 & & 1.27 & & 2.70 & & 1.80 & & 0.97 & & 0.80 & & 0.30 & \\
\hline 150 & 3.00 & & 1.80 & & 1.20 & & 1.73 & & 3.40 & & 2.20 & & 1.20 & & 1.20 & & 0.50 & \\
\hline 160 & 3.70 & & 2.50 & & 1.60 & & 2.88 & & 5.00 & & 2.80 & & 1.80 & & 2.00 & & 1.00 & \\
\hline 170 & 4.80 & & 3.70 & & 2.50 & & 5.18 & & 8.00 & & 3.80 & & 3.20 & & 3.50 & & 2.50 & \\
\hline 180 & 7.00 & & 5.50 & & 6.00 & & 11.5 & & 15.0 & & 6.00 & & 8.00 & & 8.00 & & 8.00 & \\
\hline ICS & 232.9 & & 125.1 & & 86.5 & & 72.6 & & 64.3 & & 43.4 & & 31.8 & & 22.7 & & 13.3 & \\
\hline MTCS & 36.6 & 5.3 & 16.2 & 2.5 & 12.8 & 2.0 & 17.2 & 2.7 & 28.5 & 4.5 & 18.7 & 3.0 & 10.4 & 1.6 & 7.2 & 1.1 & 3.3 & 0.5 \\
\hline
\end{tabular}

Table II. We present there both our uncorrected SMC results and those obtained from the dipole-Born correction procedure just described. As can be seen, the effects of this correction are dramatic at the lowest collision energies, while at higher energies they are only significant in the extreme forward direction. With the correction included, we obtain rather good agreement between measurement and calculation down to $1 \mathrm{eV}$, the lowest energy considered. However, at $10 \mathrm{eV}$ and above, there are significant quantitative differences between the calculated and measured DCSs at intermediate scattering angles.

Differential elastic cross sections measured at a fixed scattering angle as a function of the collision energy, sometimes referred to as "elastic excitation functions," are shown in Fig. 3. Such cross sections provide stringent tests of the agreement between computation and experiment and may reveal resonant features not apparent in DCS data obtained at fixed energies. In the present case, the experimental results of Fig. 3 show no clear evidence of the low-energy $\pi^{*}$ resonance; at most, there is some suggestion of an enhancement between roughly 1 and $1.5 \mathrm{eV}$. In their high-resolution study, Benoit and coworkers [11] observed resonant peaks with superposed "boomerang" oscillations [65] in the excitation cross sections for several vibrational modes but stated that the elastic cross section was "structureless." Although it is unclear whether they were referring to a lack of boomerang structure or the absence of a resonant peak, in the closely related formic acid molecule, Allan [39] found that the $\pi^{*}$ resonance produced only a weak shoulder in the vibrationally elastic cross section, as opposed to peaks in the vibrational-excitation channels. In any case, it appears that the acetaldehyde $\pi^{*}$ resonance is either absent from our measurements or too weak and broad to stand out clearly against the strong dipole-scattering background. On the other hand, it stands out clearly in our calculated cross sections (without Born correction) at $90^{\circ}$ and $125^{\circ}$. However, calculations such as ours that do not consider vibrational motion invariably yield resonances that are too narrow and strong, both because they neglect vibrational broadening and because they preclude loss of flux to vibrationally inelastic channels; thus, we do not expect to observe such a sharp peak in the measured data. Although the sharp peak is absent from the Born-corrected cross sections shown in Fig. 3, we consider the resulting agreement with the measurements somewhat fortuitous: being $\pi^{*}$ in character, the resonance is predominantly associated with the $\ell=2$ partial wave, and in this energy range, the $\ell=2$ contribution to the corrected results was taken from the (necessarily nonresonant) dipole-Born amplitude.

At higher energies, the fixed-angle measurements of Fig. 3 show broad features whose number and position depend on the scattering angle. At $55^{\circ}$, there is a plateau running from about 5 to $12 \mathrm{eV}$ that may result from overlapping broad peaks; at $90^{\circ}$, there is a shoulder at about $4 \mathrm{eV}$ and a peak at about $7 \mathrm{eV}$; while at $125^{\circ}$, there is a single pronounced peak centered at about $8 \mathrm{eV}$. The last two of these may plausibly be associated with the broad maximum centered at $6.8 \mathrm{eV}$ seen by Dressler 

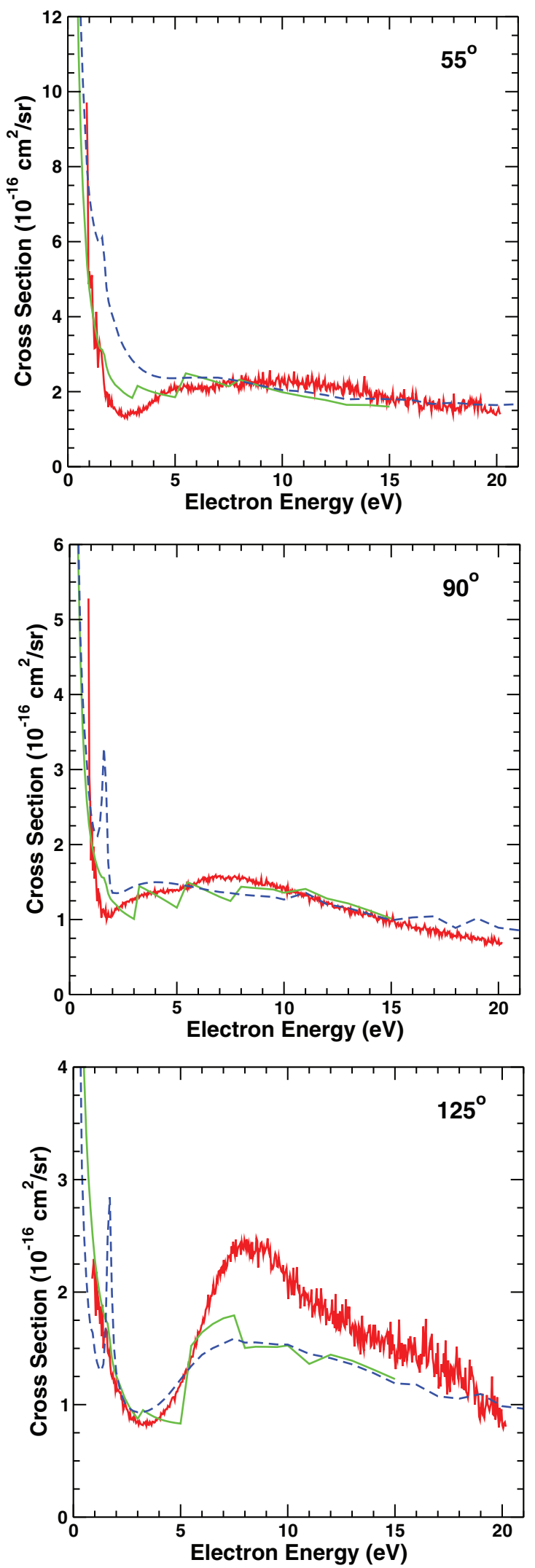

FIG. 3. (Color online) Differential cross sections for elastic electron scattering by acetaldehyde at the fixed scattering angles indicated in each panel. The red line is the present measurement, the blue dashed line the present calculation, and the green solid line the present calculation including the Born correction.

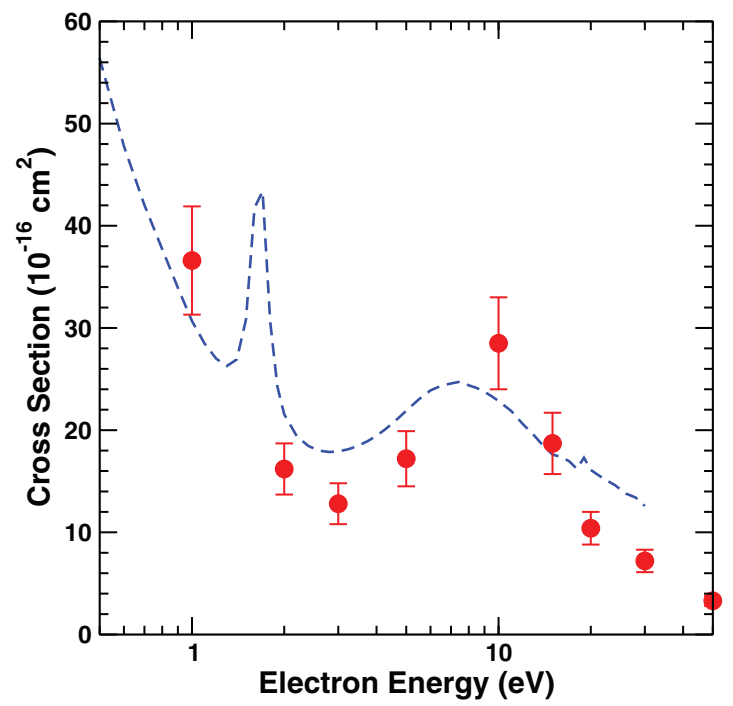

FIG. 4. (Color online) Momentum-transfer cross section for elastic electron scattering by acetaldehyde. The red circles are the present measurements, and the dashed blue line is the present calculation.

and Allan [10] in the vibrational-excitation cross section and attributed by them to one or more $\sigma^{*}$ shape resonances.

Above the energy of the $\pi^{*}$ resonance, the dipole-corrected DCSs display discontinuities where the value of $L_{\max }$ changes but otherwise tend to track the uncorrected results, which is not surprising inasmuch as the influence of the dipole decreases with increasing energy and scattering angle. The overall agreement between the measured and calculated results in Fig. 3 is only fair. At $55^{\circ}$ and $90^{\circ}$, the calculated DCS is in semiquantitative agreement with the measurements but appears to miss the $7-\mathrm{eV}$ peak seen in the measured $90^{\circ} \mathrm{DCS}$; at $125^{\circ}$, the qualitative agreement is rather good, but the $8-\mathrm{eV}$ peak is much more pronounced in the measured than in the calculated cross section.

Our momentum-transfer cross section (MTCS) and integral elastic cross section are shown in Figs. 4 and 5, and the experimental values are also listed in Table II. For the MTCS, we show only uncorrected results because the dipole-Born correction is most significant at forward angles and thus has only a minor effect on the MTCS. As might be expected from the DCSs just presented, agreement between the measured and calculated results for the MTCS is only fair. Both show a broad peak between 5 and $15 \mathrm{eV}$, but the measurements indicate a higher maximum value. At $20 \mathrm{eV}$ and above, meanwhile, the computed cross section exceeds the measurements, likely because the single-channel calculation precludes loss of flux to electronically inelastic channels.

As seen in Fig. 5, the dipole-Born correction greatly increases both the ICS values obtained from the measurements and those obtained from the SMC calculation. As was the case for the MTCS, the experimental ICS evaluated without the dipole-Born correction for forward scattering is in fair agreement with the uncorrected SMC ICS. The corrected values are also in fair agreement, although the SMC result is generally larger. As noted in Sec. III, the Hartree-Fock dipole moment, which for consistency was used to evaluate the Born correction to the SMC cross section, is $22 \%$ larger 


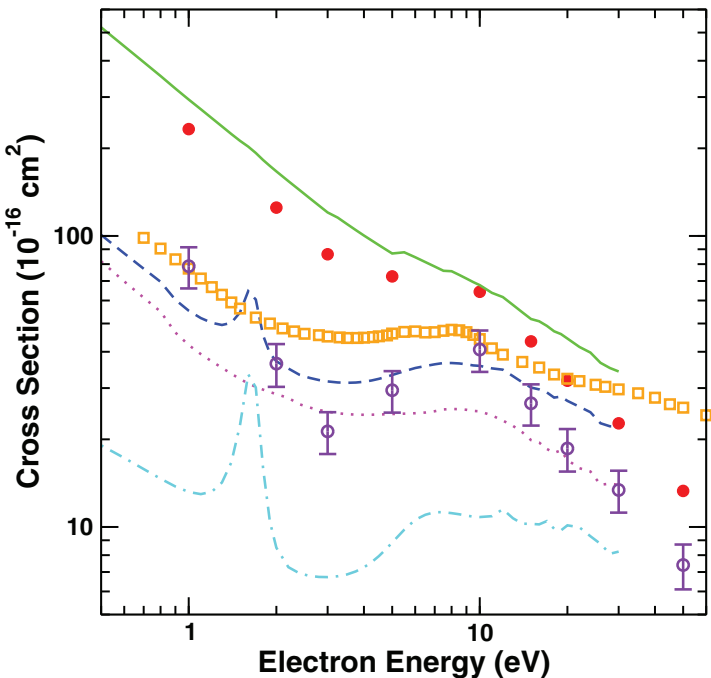

FIG. 5. (Color online) Integral cross section for elastic scattering of low-energy electrons by acetaldehyde. The red circles are the present measurements, and the solid green line is the present calculation, both with a dipole-Born correction for long-range scattering. The open violet circles show the ICS evaluated from the measurements without a dipole-Born correction, while the dashed blue line shows the uncorrected SMC calculation, and the dotted magenta and chained cyan lines are, respectively, its ${ }^{2} A^{\prime}$ and ${ }^{2} A^{\prime \prime}$ components. For comparison, the total scattering cross section measured by Szmytkowski [24] is shown by the orange squares.

than the experimental value, and the Born cross section scales with the square of the dipole moment. We estimate the error in the corrected SMC ICS due to the error in the dipole moment as $\sim 40 \%$ at $1 \mathrm{eV}$ and $14 \%$ at $30 \mathrm{eV}$. The effect of this error is partially offset by the different energy-loss values assumed in extrapolating the SMC and experimental DCSs to $0^{\circ}$ (10 and $5 \mathrm{meV}$, respectively). Clearly, both results are subject to considerable systematic uncertainties, but together they should provide a better representation of the true ICS than the uncorrected values. It is thus noteworthy that both are larger than the reported TCS [24] at energies up to $15 \mathrm{eV}$. Attenuation measurements on strongly polar systems such as acetaldehyde systematically underestimate the TCS, due to the impossibility of discriminating between unscattered electrons and those scattered elastically through small enough an angle to pass through the exit aperture. However, Szmytkowski [24] estimates this systematic effect at only $3 \%$ over most of the range shown in Fig. 5 and 5\% to $7 \%$ below $3 \mathrm{eV}$, much too small to account for the inconsistency seen in Fig. 5 between the reported TCS and the dipole-corrected elastic ICS results, especially at low energies. Further investigation of both the total and elastic cross sections might help clarify this discrepancy.
The decomposition of the uncorrected SMC ICS into its ${ }^{2} A^{\prime}$ and ${ }^{2} A^{\prime \prime}$ components is also shown in Fig. 5. The narrow low-energy peak is seen to occur in ${ }^{2} A^{\prime \prime}$, as expected for a $\pi^{*}$ resonance; its energy, about $1.65 \mathrm{eV}$, is somewhat above the experimental position of 1.2 to $1.3 \mathrm{eV}$ [8-11]. The broad maximum in the ICS near $10 \mathrm{eV}$ results from overlapping ${ }^{2} A^{\prime}$ and ${ }^{2} A^{\prime \prime}$ features, each of which may contain more than one broad resonance.

The isoelectronic molecule formamide also exhibits a lowenergy $\pi^{*}$ resonance. Electron transmission measurements [25] place this resonance somewhat higher in energy $(2.05 \mathrm{eV})$ than the resonance in acetaldehyde. There is less evidence for higher-energy shape resonances, however. The available calculations of the formamide elastic ICS show either a plateau [29] or weak ${ }^{2} A^{\prime}$ and ${ }^{2} A^{\prime \prime}$ maxima [30] in the 5 to $10 \mathrm{eV}$ energy range, in contrast to the pronounced peaks seen in the present calculated and measured results for acetaldehyde. Formic acid, also isoelectronic with acetaldehyde, exhibits a well-studied $\pi^{*}$ resonance at $1.8 \mathrm{eV}$ (e.g., Ref. [7]). Again, though, the calculated elastic cross sections [41-43,45] do not provide clear evidence of higher-lying $\sigma^{*}$ resonances, while the available elastic measurements [36] lack the energy resolution to indicate whether such features are present. There is, however, a broad maximum in the measured TCS between roughly 5 and $15 \mathrm{eV}$ [32].

\section{SUMMARY}

We have presented computed and experimental cross sections for the elastic scattering of low-energy electrons by acetaldehyde. The calculated and measured DCSs are in reasonable agreement from 1 to $15 \mathrm{eV}$, although the measurements show stronger high-angle scattering at energies around $10 \mathrm{eV}$. The $\mathrm{C}-\mathrm{O} \pi^{*}$ shape resonance that is seen in electron-transmission $[8,9]$ and vibrational-excitation $[10,11]$ measurements is also seen in our fixed-nuclei elastic calculations; however, it is not apparent in either our measured elastic cross sections or the total cross section of Ref. [24]. A broad maximum near $8 \mathrm{eV}$ appears to result from overlapping resonances of both ${ }^{2} A^{\prime}$ and ${ }^{2} A^{\prime \prime}$ symmetry.

\section{ACKNOWLEDGMENTS}

This work was sponsored by the US National Science Foundation under Grants No. PHY-0968873 and No. PHY-0968874. The work of V.M. and C.W. was supported by the Chemical Sciences, Geosciences and Biosciences Divsion, Office of Basic Energy Sciences, Office of Science, US Department of Energy under Grant No. DE-FG02-97ER14814 and made use of the Jet Propulsion Laboratory's Supercomputing and Visualization Facility.
[1] C. A. Gottlieb, in Molecules in the Galactic Environment, edited by M. A. Gordon and L. E. Snyder (Wiley-Interscience, New York, 1973), pp. 181-186.

[2] N. Fourikis, M. W. Sinclair, B. J. Robinson, P. D. Godfrey, and R. D. Brown, Aust. J. Phys. 27, 425 (1974).
[3] W. Gilmore, M. Morris, D. R. Johnson, F. J. Lovas, B. Zuckerman, B. E. Turner, and P. Palmer, Astrophys. J. 204, 43 (1976).

[4] G. A. Gallup, P. D. Burrow, and I. I. Fabrikant, Phys. Rev. A 79, 042701 (2009). 
[5] T. N. Rescigno, C. S. Trevisan, and A. E. Orel, Phys. Rev. A 80, 046701 (2009).

[6] G. A. Gallup, P. D. Burrow, and I. I. Fabrikant, Phys. Rev. A 80, 046702 (2009).

[7] R. Janečková, D. Kubala, O. May, J. Fedor, and M. Allan, Phys. Rev. Lett. 111, 213201 (2013).

[8] E. H. van Veen, W. L. van Dijk, and H. H. Brongersma, Chem. Phys. 16, 337 (1976).

[9] K. D. Jordan and P. D. Burrow, Acc. Chem. Res. 11, 341 (1978).

[10] R. Dressler and M. Allan, J. Electron Spectrosc. Relat. Phenom. 41, 275 (1986).

[11] C. Benoit, R. Abouaf, and S. Cvejanovic, Chem. Phys. 117, 295 (1987).

[12] W. T. Naff, R. N. Compton, and C. D. Cooper, J. Chem. Phys. 57, 1303 (1972).

[13] W.-C. Tam and C. E. Brion, J. Electron Spectrosc. Relat. Phenom. 3, 467 (1974).

[14] R. H. Staley, L. B. Harding, W. A. Goddard, III, and J. L. Beauchamp, Chem. Phys. Lett. 36, 589 (1975).

[15] T. Ari and J. B. Hasted, Chem. Phys. Lett. 85, 153 (1982).

[16] K. N. Walzl, C. F. Koerting, and A. Kuppermann, J. Chem. Phys. 87, 3796 (1987).

[17] F. H. Dorman, J. Chem. Phys. 44, 3856 (1966).

[18] R. Dressler and M. Allan, Chem. Phys. Lett. 118, 93 (1985).

[19] E. Szymańska, V. S. Prabhudesai, N. J. Mason, and E. Krishnakumar, Phys. Chem. Chem. Phys. 15, 998 (2013).

[20] F. H. Dorman, J. Chem. Phys. 42, 65 (1965).

[21] K. Głuch, J. Cytawa, and L. Michalak, Int. J. Mass Spectrom. 273, 20 (2008).

[22] J. N. Bull and P. W. Harland, Int. J. Mass Spectrom. 273, 53 (2008).

[23] E. Burean and P. Swiderek, J. Phys. Chem. C 112, 19456 (2008).

[24] C. Szmytkowski, J. Phys. B 43, 055201 (2010).

[25] M. Seydou, A. Modelli, B. Lucas, K. Konate, C. Desfrançois, and J. P. Schermann, Eur. Phys. J. D 35, 199 (2005).

[26] F. F. da Silva, S. Denifl, T. D. Märk, N. L. Doltsinis, A. M. Ellis, and P. Scheier, J. Phys. Chem. A 114, 1633 (2010).

[27] T. Hamann, A. Edtbauer, F. F. da Silva, S. Denifl, P. Scheier, and P. Swiderek, Phys. Chem. Chem. Phys. 13, 12305 (2011).

[28] T. P. M. Goumans, F. A. Gianturco, F. Sebastianelli, I. Baccarelli, and J. L. Rivail, J. Chem. Theor. Comp. 5, 217 (2009).

[29] M. H. F. Bettega, Phys. Rev. A 81, 062717 (2010).

[30] Y.-F. Wang and S. X. Tian, Phys. Rev. A 85, 012706 (2012).

[31] J. B. Maljković, F. Blanco, G. García, B. P. Marinković, and A. R. Milosavljević, Nucl. Instrum. Methods Phys. Res., Sect. B 279, 124 (2012).

[32] M. Kimura, O. Sueoka, A. Hamada, and Y. Itikawa, Adv. Chem. Phys. 111, 537 (2000).

[33] K. Aflatooni, B. Hitt, G. A. Gallup, and P. D. Burrow, J. Chem. Phys. 115, 6489 (2001).

[34] A. Pelc, W. Sailer, P. Scheier, N. J. Mason, and T. D. Märk, Eur. Phys. J. D 20, 441 (2002).

[35] A. Pelc, W. Sailer, P. Scheier, M. Probst, N. J. Mason, E. Illenberger, and T. D. Märk, Chem. Phys. Lett. 361, 277 (2002).
[36] V. Vizcaino, M. Jelisavcic, J. P. Sullivan, and S. J. Buckman, New J. Phys. 8, 85 (2006).

[37] V. S. Prabhudesai, D. Nandi, A. H. Kelkar, R. Parajuli, and E. Krishnakumar, Chem. Phys. Lett. 405, 172 (2005).

[38] M. Allan, J. Phys. B 39, 2939 (2006).

[39] M. Allan, Phys. Rev. Lett. 98, 123201 (2007).

[40] D. Kubala, O. May, and M. Allan, J. Phys.: Conf. Ser. 388, 052016 (2012).

[41] F. A. Gianturco and R. R. Lucchese, New J. Phys. 6, 66 (2004).

[42] F. A. Gianturco and R. R. Lucchese, Eur. Phys. J. D 39, 399 (2006).

[43] C. S. Trevisan, A. E. Orel, and T. N. Rescigno, Phys. Rev. A 74, 042716 (2006).

[44] C. S. Trevisan, A. E. Orel, and T. N. Rescigno, Elastic Electron Scattering from Formic Acid, Technical Report LBNL-62280 (Lawrence Berkeley National Laboratory, Berkeley, CA, 2008).

[45] M. H. F. Bettega, Phys. Rev. A 74, 054701 (2006).

[46] M. A. Khakoo, C. E. Beckmann, S. Trajmar, and G. Csanak, J. Phys. B 27, 3159 (1994).

[47] J. H. Brunt, G. C. King, and F. H. Read, J. Phys. B 10, 1289 (1977).

[48] M. A. Khakoo, K. Keane, C. Campbell, N. Guzman, and K. Hazlett, J. Phys. B 40, 3601 (2007).

[49] M. Hughes, J. K. E. James, J. G. Childers, and M. A. Khakoo, Meas. Sci. Technol. 14, 841 (1994).

[50] R. K. Nesbet, Phys. Rev. A 20, 58 (1979).

[51] D. R. Lide, Editor, CRC Handbook of Chemistry and Physics, 76th ed. (CRC Press, Boca Raton, FL, 1995), p. 337.

[52] K. Takatsuka and V. McKoy, Phys. Rev. A 24, 2473 (1981).

[53] K. Takatsuka and V. McKoy, Phys. Rev. A 30, 1734 (1984).

[54] C. Winstead and V. McKoy, Adv. At. Mol. Opt. Phys. 36, 183 (1996).

[55] C. Winstead and V. McKoy, Comput. Phys. Commun. 128, 386 (2000).

[56] R. Ditchfield, W. J. Hehre, and J. A. Pople, J. Chem. Phys. 54, 724 (1971).

[57] W. J. Hehre, R. Ditchfield, and J. A. Pople, J. Chem. Phys. 56, 2257 (1972).

[58] M. W. Schmidt, K. K. Baldridge, J. A. Boatz, S. T. Elbert, M. S. Gordon, J. H. Jensen, S. Koseki, N. Matsunaga, K. A. Nguyen, S. Su, T. L. Windus, M. Dupuis, and J. A. Montgomery, Jr, J. Comput. Chem. 14, 1347 (1993).

[59] T. H. Dunning, Jr., J. Chem. Phys. 90, 1007 (1989).

[60] R. A. Kendall, T. H. Dunning, Jr., and R. J. Harrison, J. Chem. Phys. 96, 6796 (1992).

[61] P. H. Turner and A. P. Cox, J. Chem. Soc. Faraday Trans. 2 74, 533 (1978).

[62] C. W. Bauschlicher, J. Chem. Phys. 72, 880 (1980).

[63] C. Winstead, V. McKoy, and M. H. F. Bettega, Phys. Rev. A 72, 042721 (2005).

[64] T. N. Rescigno and B. I. Schneider, Phys. Rev. A 45, 2894 (1992).

[65] A. Herzenberg, J. Phys. B 1, 548 (1968). 